\title{
Control predictivo/adaptativo de sistemas complejos utilizando técnicas de ingeniería neuronal*
}

\author{
José Gallardo** \\ Claudio Ayala** \\ Rubén Castro ${ }^{* * * *}$
}

\author{
Recibido: 24/10/2017 • Aceptado: 15/03/2018 \\ https://doi.org/10.22395/rium.v17n33a8
}

\begin{abstract}
Resumen
Se presenta el diseño e implementación de un sistema de control predictivo/adaptativo, utilizando técnicas de ingeniería neuronal para controlar un sistema MIMO no lineal con el objeto de controlar, en una etapa posterior, la temperatura y el nivel en una planta no lineal de tipo cónica. Preliminarmente, se ensayaron estructuras de control convencional lo que hizo surgir la necesidad de probar estructuras de control inteligente que permitan cumplir más eficazmente con los objetivos de control. El proceso se inicia con la experimentación de diferentes estructuras de control neuronal, para luego escalar hacia un sistema de control neuronal predictivo/adaptativo. Los resultados logrados a nivel simulación, ensayando el diseño propuesto sobre modelos matemáticos de sistemas MIMO no lineales, fueron satisfactorios y cumplieron los objetivos de control establecidos, por tanto, en la siguiente etapa del proyecto, se estima la experimentación en la planta real en estudio.
\end{abstract}

Palabras clave: ingeniería neuronal; identificación; control predictivo; control adaptativo; sistemas MIMO no lineales.

El artículo se origina en una investigación conducente al título de maestría titulada Diseño e implementación de sistema de control para estanque cónico mediante redes neuronales, que se ejecutó de marzo de 2016 a diciembre de 2017.

** Ph. D. Académico de la Universidad Católica del Norte, Facultad de Ingeniería, Departamento de Ingeniería de Sistemas y Computación. Av. Angamos 0610, Antofagasta, Chile. Teléfono: +56 55 2355157, jgallardo@ucn. cl. Orcid: https://orcid.org/0000-0002-6477-5302

*** $\quad \mathrm{Ph}$. D. Académico de la Universidad de Antofagasta, Facultad de Ingeniería, Departamento de Ingeniería Eléctrica. Av. Angamos 0601, Antofagasta, Chile. Teléfono: +56 55 2637474, claudio.ayala@uantof.cl.

**** Académico de la Universidad Arturo Prat, Facultad de Ingeniería y Arquitectura, Av. Arturo Prat 2120, Iquique, Chile. Teléfono (+56) 57 2526456, rucastro@unap.cl. Orcid: https://orcid.org/0000-0002-7613-1301 


\title{
Predictive/Adaptive Control of Complex Systems Using Neural Engineering Techniques
}

\begin{abstract}
The design and implementation of a predictive/adaptive control system is presented, using neural engineering techniques to control a non-linear MIMO system in order to control, at a later stage, the temperature and level in a non-linear conical plant. Preliminarily, conventional control structures were tested, which gave rise to the need to test intelligent control structures that allow the control objectives to be met more effectively. The process begins with the experimentation of different neuronal control structures, and then escalates to a predictive/adaptive neuronal control system. The results achieved at the simulation level, testing the proposed design on mathematical models of non-linear MIMO systems, were satisfactory and met the control objectives established, therefore, in the next stage of the project, the experimentation is estimated in the real plant under study.
\end{abstract}

Keywords: neuronal engineering; identification; predictive control; adaptive control; non-linear MIMO systems.

\section{Controle preditivo/adaptativo de sistemas complexos utilizando técnicas de engenharia neural}

\section{Resumo}

Apresenta-se a criação e a implementação de um sistema de controle preditivo/adaptativo utilizando técnicas de engenharia neural para controlar um sistema MIMO não linear com o objetivo de controlar, em uma etapa posterior, a temperatura e o nível em uma planta não linear de tipo cônica. Preliminarmente, estruturas de controle convencional foram ensaiadas, o que fez com que surgisse a necessidade de testar estruturas e controle inteligente que permitissem cumprir os objetivos de controle de forma mais eficaz. O processo começa com a experimentação de diferentes estruturas de controle neural, para depois escalar em direção a um sistema de controle neural preditivo/adaptativo. Os resultados alcançados na simulação, ensaiando o desenho proposto sobre modelos matemáticos de sistemas MIMO não lineares, foram satisfatórios e cumpriram os objetivos de controle estabelecidos, portanto, na seguinte etapa do projeto, estima-se realizar a experimentação na planta real em estudo.

Palavras-chave: engenharia neural; identificação; controle preditivo; controle adaptativa; sistemas MIMO não lineares. 


\section{INTRODUCCIÓN}

En el escenario de globalización actual, la competencia en muchos sectores industriales, así como el marcado interés social por los problemas medioambientales relacionados con los procesos de producción, impulsa la necesidad de disponer de técnicas fiables y flexibles que permitan la operación de un proceso con gran eficiencia. Por lo tanto, el objetivo de todo sistema de control deberá ser actuar sobre las variables manipuladas de forma que puedan satisfacerse múltiples y cambiantes criterios de funcionamiento (beneficio económico, seguridad del proceso y del personal, problemas medioambientales, etc.), propios de los sistemas complejos y en presencia de cambios en las características del proceso.

Sin embargo, el controlar procesos complejos no es una tarea simple debido a que estos son frecuentemente no lineales e inciertos y el derivar un modelo matemático del proceso sobre el cual el diseño del controlador será basado, es una tarea más compleja, por lo tanto, es de gran relevancia el utilizar sofisticados y fiables sistemas de control [12, 18].

El trabajo que aquí se presenta, reporta una investigación en la cual se requería controlar la temperatura y el nivel de líquido en un estanque cónico (no lineal). En un principio se desarrollaron algunos esquemas de control convencional (basados en controladores PID) para enfrentar el problema planteado, sin embargo, no se lograron los resultados esperados, razón por la cual se recurrió a esquemas de control avanzado, que permiten resolver el problema cumpliendo más eficientemente los objetivos de control. Al respecto, es posible encontrar en la literatura múltiples estrategias de control avanzado que han sido propuestas [9] tales como el control de ganancia programada, el control predictivo basado en modelos matemáticos, el control experto, el control neuronal, el control Fuzzy, etc. De todas estas estrategias desarrolladas, las técnicas de control neuronal y las de control predictivo parecen ser las más eficientes y, consecuentemente, las que más amplia aceptación han logrado en la industria por cuanto, en su forma más general, aceptan cualquier tipo de modelos y funciones objetivo o restricciones. Algunas aplicaciones exitosas reportadas en la literatura son: en [16] se estudia el control de flujo en estanques industriales, en [14] se implementan métodos de control inteligente usando procedimientos de agrupación y clasificación mediante redes neuronales y en [1] se aplica neurocontrol para el control de una planta de destilación.

A continuación, en la segunda sección de este texto, se presentan las diversas estructuras de neurocontrol ensayadas al inicio de la investigación, la tercera sección presenta el diseño y desarrollo de un sistema de control predictivo/adaptativo para aplicarse a futuro en la planta en estudio, la cuarta sección presenta modelos matemáticos de algunas plantas, para validar en modo de simulación la efectividad del sistema de control que se propone, finalmente, en las secciones quinta y sexta, se presentan los resultados logrados, conclusiones finales y trabajo futuro respectivamente. 


\section{ESTRUCTURAS DE NEUROCONTROL EVALUADAS}

Para poder sintetizar una arquitectura adecuada, se realizó, en principio, el estudio de diversos modelos de control neuronal que han sido propuestos en distintos artículos $[8,11,15,17]$, los que fueron ensayados con el propósito de proyectar una futura estrategia de control.

Las configuraciones de control ensayadas se muestran en las figuras 1, 2, 3 y 4 .

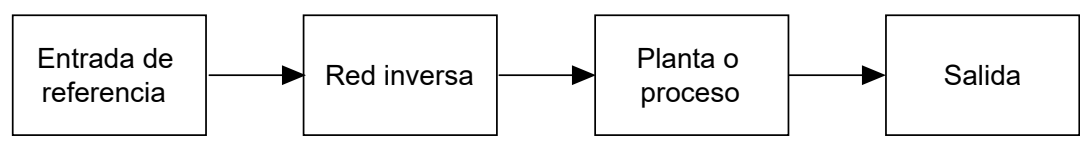

Figura 1. Control basado en el modelo inverso.

Fuente: elaboración propia.

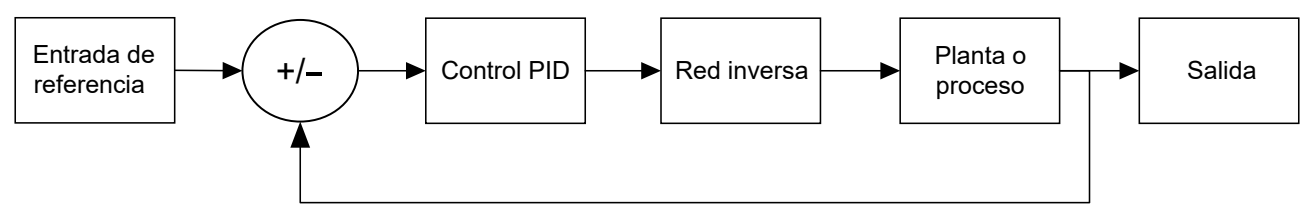

Figura 2. Control con modelo inverso en el lazo.

Fuente: elaboración propia.

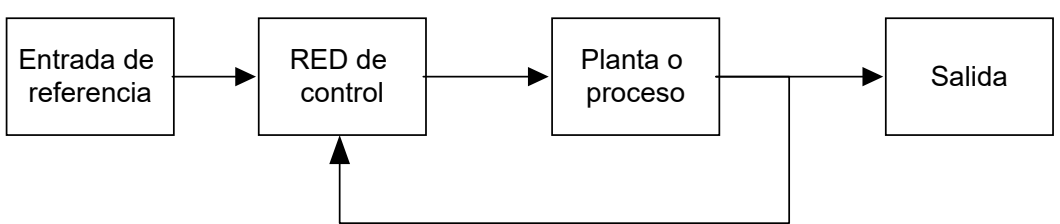

Figura 3. Control directo sin uso de modelo

Fuente: elaboración propia.

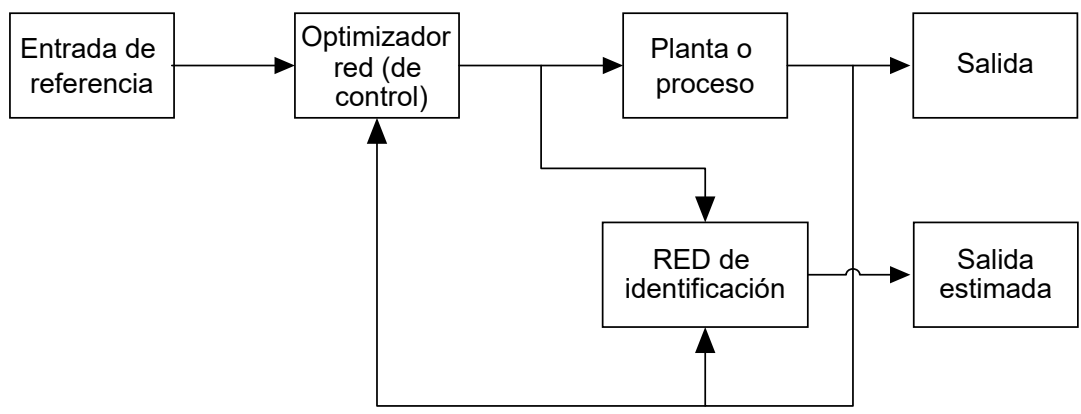

Figura 4. Control directo con uso de modelo

Fuente: elaboración propia 
- Primer esquema de control: modelo inverso.

- Segundo esquema de control: modelo inverso en el lazo.

- Tercer esquema de control: esquema directo sin uso de modelo.

- Cuarto esquema de control: esquema directo con uso de modelo.

El estudio y ensayo de los modelos de control citados, permitió evaluar y constatar que los esquemas de redes neuronales para la obtención de modelos directo e inverso de procesos no lineales, son bastante eficaces, como así mismo estructuras en las cuales la red actúa como un controlador propiamente tal. Los esquemas de control fueron ensayados a nivel de simulación en el ambiente simulink de Matlab.

\section{DISEÑO Y DESARROLLO DE UN SISTEMA DE UN CONTROL PREDICTIVO/ADAPTATIVO}

En consideración de la experiencia lograda y los promisorios resultados obtenidos en la sección anterior, se decidió diseñar un sistema que permitiera controlar eficientemente un sistema MIMO no lineal, el cual se basa en el esquema general presentado en la figura 5. En este esquema se pueden identificar esencialmente tres elementos: un predictor, el cual es un modelo del proceso que se desea controlar y que permite predecir las futuras salidas del proceso en un determinado horizonte de predicción, el controlador, cuya función es generar las señales de control óptimas mediante la minimización de la función de coste definida y la planta a ser controlada. La mayoría de los sistemas de control predictivo desarrollados, se basan fundamentalmente en este diagrama general, aunque presentan diferencias en el algoritmo utilizado por el optimizador para minimizar la función de coste definida y el método utilizado para generar el predictor, el cual es un modelo del proceso que se desea controlar.

Considerando, que el problema de diseño del sistema de control a desarrollar se basa fundamentalmente en la obtención de un modelo de predicción y en el diseño del controlador, a continuación, se muestra por separado el diseño de ambos módulos.

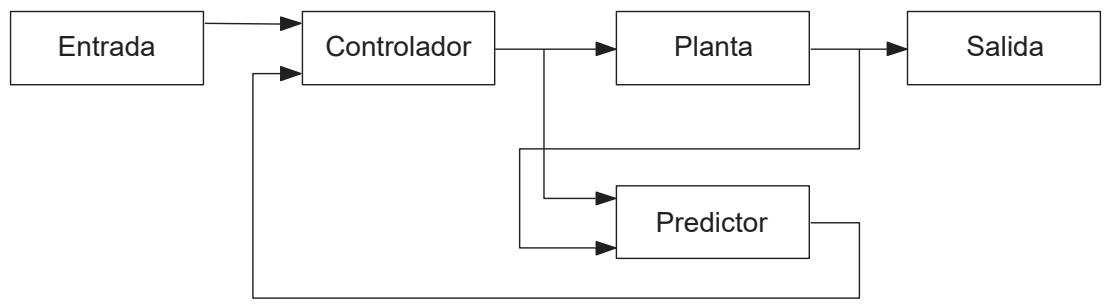

Figura 5. Esquema general del sistema

Fuente: elaboración propia 


\subsection{Modelo de predicción}

Si se considera que obtener un modelo que represente la dinámica de un sistema es muy importante para diseñar un sistema de control que cumpla eficazmente los objetivos de control y que este proceso no es trivial, se ha optado por aprovechar las bondades que presentan las redes neuronales para dicho propósito. Pionero en la investigación sobre identificación y control de sistemas por medio de redes neuronales ha sido K. S. Narendra, quien describe en [8], cómo la dinámica de sistemas no lineales puede ser modelada mediante redes neuronales. Tanto redes neuronales de tipo feedforward como redes recurrentes [13], se recomiendan para la identificación de sistemas dinámicos. Para el presente trabajo, se utilizarán las redes feedforward multicapa donde la dinámica del sistema será representada por ventanas temporales pasadas, tanto de entradas como de salidas del sistema. Las redes neuronales recurrentes no requieren del uso de señales retardadas pues tienen dinámica propia.

La identificación de sistemas mediante redes neuronales puede realizarse con entrenamiento off-line (fuera de línea) o con entrenamiento on-line (en línea). Mucha investigación se ha efectuado sobre la identificación de sistemas off-line donde se usa un archivo generado con la historia de las entradas y salidas del sistema para entrenar la red [11], sin embargo, para propósitos de control adaptivo, los algoritmos de entrenamiento on-line son requeridos para proveer una notable mejoría en la exactitud del sistema modelado y ajustar los parámetros de la red de acuerdo a los cambios que ocurran en el proceso.

Un modelo llamado de entrada-salida, describe la dinámica del sistema, basado en datos de entrada y salida. Este modelo puede ser un modelo del tipo Narmax [3] y asume que la nueva salida del sistema, puede ser estimada desde sus entradas y salidas pasadas. Narendra, en [8], plantea otras tres clases de modelos para caracterizar una planta, sin embargo, el modelo llamado de entrada-salida descrito, es el modelo más general. El modelo de entrada-salida (figura 6), puede ser representado por la siguiente ecuación.

$$
y_{p}(k+1)=f(y(k), y(k-1), \ldots ., y(k-n) ; u(k), u(k-1), \ldots ., u(k-m))
$$

Donde $[\mathrm{u}(\mathrm{k}), \mathrm{y}(\mathrm{k})]$, representan el par de entrada salida en el tiempo k. Enteros positivos de $\mathrm{n}$ y $\mathrm{m}$ son respectivamente el número de salidas pasadas (llamado también el orden del sistema) y el número de entradas pasadas [3]. En la práctica $m$ es usualmente menor o igual a $n, f$, puede ser una función no lineal que mapea las entradas y salidas pasadas para una nueva salida. 


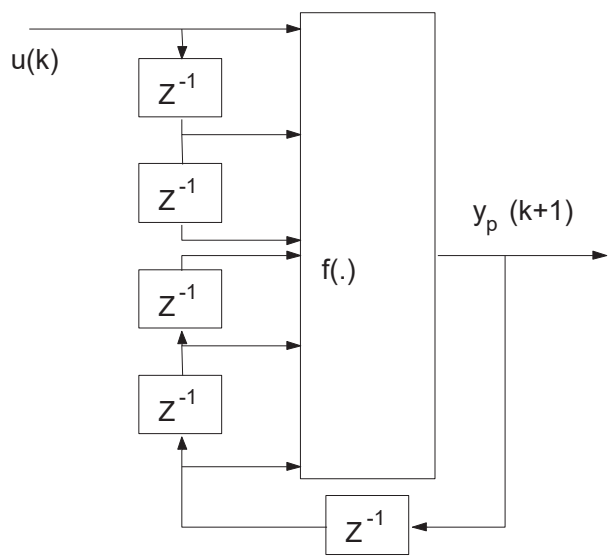

Figura 6. Esquema general del sistema

Fuente: elaboración propia

Una aproximación obvia para el sistema modelado, es elegir una estructura de red neuronal que desempeñe la misma función. Denotando por $\mathrm{y}_{\mathrm{m}}$ la salida de la red se tiene:

$$
y_{m}(k+1)=\hat{f}(y(k), y(k), \ldots . ., y(k-n) ; u(k), u(k-1), \ldots ., u(k-m))
$$

En esta expresión, $\hat{f}$ representa el mapa entrada-salida de la red, es decir la aproximación de $f$. Hay que notar que la entrada a la red contendrá los valores pasados de entrada y salida al sistema real (la red no tiene realimentación). Luego de un cierto periodo de entrenamiento, la salida de la red será aproximadamente igual a la salida real de la planta, en ese momento la red se puede alimentar a sí misma, y entonces posteriormente la red puede ser usada independientemente de la planta.

Asumiendo que redes multicapa feedforward, con al menos una capa oculta, tienen la capacidad de aproximar bastante bien cualquier función no lineal, se ha elegido una clásica red backpropagation con una capa oculta de diez neuronas, funciones de activación tangente hiperbólica en la capa oculta y lineal en la capa de salida para representar el proceso a controlar. De esta forma la red que asume el papel de predictor puede ser representado por la siguiente ecuación:

$$
y_{p}(t+1)=w_{2}\left[\tanh \left(w_{1} p+b_{1}\right)\right]+b_{2}
$$

Donde $\mathrm{w}_{1}, \mathrm{w}_{2}, \mathrm{~b}_{1} \mathrm{y} \mathrm{b}_{2}$ son las matrices de pesos y bias y $p$ es el vector de entrada a la red.

$$
p=[y(t), y(t-1), \ldots ., y(t-n), u(t), u(t-1), \ldots ., u(t-m)]
$$




\subsection{Controlador}

El controlador está constituido por un optimizador como controlador primario y una red neuronal controladora como controlador secundario. Esta red controladora se conecta en paralelo con el optimizador y una vez entrenada, puede constituirse en el controlador primario. Esta configuración otorga una mayor robustez al sistema desarrollado y aliviana la carga de cómputo del optimizador con idéntico desempeño.

\section{Optimizador}

El optimizador cuya misión es minimizar la función objetivo definida, puede ser implementado utilizando alguno de los siguientes métodos: el método de gradiente descendente, el método de Newton, los métodos cuasi-Newton o una red neuronal. Para este desarrollo, se ha optado por utilizar una variante [2] del método del gradiente descendente el cual está definido por la siguiente ecuación.

$$
u(t+1)=u(t)-\eta \frac{\partial J}{\partial u(t)}+\alpha(u(t)-u(t-1))
$$

Donde $u(t)$, es la señal de control, $\eta$ es la llamada ganancia, $\alpha$ el momento y $J$ es la función objetivo a minimizar. Si se requiere que la salida sea lo más próxima posible a la referencia y no se consideran restricciones en las variables, se puede definir la función objetivo de acuerdo a la siguiente expresión:

$$
J=\frac{1}{2} e^{2}(t+1)
$$

Aquí, $e(t+1)=r(t+1)-y_{p}(t+1)$, es la señal de error futuro, entre la señal de referencia y la salida del modelo de predicción.

El gradiente de la función objetivo puede ser calculado explícitamente desde el modelo neuronal del proceso. Así:

$$
\frac{\partial J}{\partial u(t)}=-e(t+1) \frac{\partial y_{p}(t+1)}{\partial u(t)}
$$

Considerando que:

$$
\frac{\partial y_{p}(t+1)}{\partial u(t)}=w_{2}\left[\operatorname{sech}^{2}\left(w_{1} p+b_{1}\right)\right] w_{1} \frac{d p}{d u(t)}
$$

Donde: $\frac{d p}{d u(t)}=[0,0, \ldots, 0,1,0, \ldots ., 0]$ 
Para un horizonte de predicción igual a $T$ se debe tener en cuenta el cálculo de las futuras señales de control [2]. Por esta razón, es necesario no solo considerar los errores presentes entre la referencia y la salida del modelo de predicción, sino también la predicción de los errores futuros. Para estos efectos, se deben calcular las salidas futuras del proceso desde el modelo neuronal de predicción y las futuras señales de referencia. Así, el vector de errores quedará definido por:

$$
E_{t, T}=[e(t+1), e(t+2), \ldots \ldots \ldots, e(t+T)]
$$

Donde:

$$
e(t+i)=r(t+i)-y_{p}(t+i)
$$

Y el vector de señales futuras de control por:

$$
U_{t, T}=[u(t+1), u(t+2), \ldots \ldots ., u(t+T)]
$$

Vector que debe minimizar la función objetivo $J$ ' definida por:

$$
J^{\prime}=\frac{1}{2}\left[E_{t, T}^{T} E_{t, T}\right]
$$

Luego

$$
U_{t, T}^{K+1}=U_{t, T}^{K}-\eta \frac{\partial J^{\prime}}{\partial U_{t, T}^{k}}+\alpha\left(U_{t, T}^{K}-U_{t, T}^{K-1}\right)
$$

Donde el gradiente de la función objetivo [2], es representado por la ecuación (13):

$$
\frac{\partial J^{\prime}}{\partial U_{t, T}^{K}}=E_{t, T} \frac{\partial Y_{p ; t, T}}{\partial U_{t, T}^{k}}
$$

Y la matriz jacobiana expresada por:

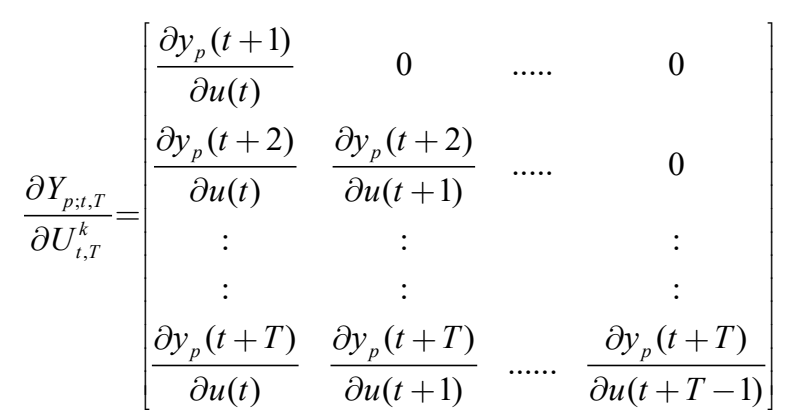




\section{Red neuronal controladora}

Como ya fue mencionado, se ha incorporado una red neuronal como controlador secundario o de respaldo para dar una mayor robustez al sistema desarrollado. La red neuronal controladora, consiste en una red backpropagation con dinámica externa. Consta de una capa oculta de 15 neuronas (aplicando el teorema de Kolmogorov [5]), función de activación tangente hiperbólica en las neuronas de la capa oculta y lineal en las neuronas de la capa de salida. Esta red neuronal tiene como objetivo mapear las entradas y salidas del optimizador. Una vez entrenada esta red (entrenamiento en línea), puede asumir el rol de controlador primario con igual desempeño al del optimizador, reduciendo ostensiblemente la cantidad de cómputo de este último. El sistema desarrollado se ilustra en la figura 7.

Por último, para reducir el offset en la salida debido a modelos de predicción no suficientemente exactos, se decidió adicionar a la señal de control un integrador que comúnmente es usado como un controlador realimentado. El esquema final del sistema de control diseñado y experimentado se muestra en la figura 8 .

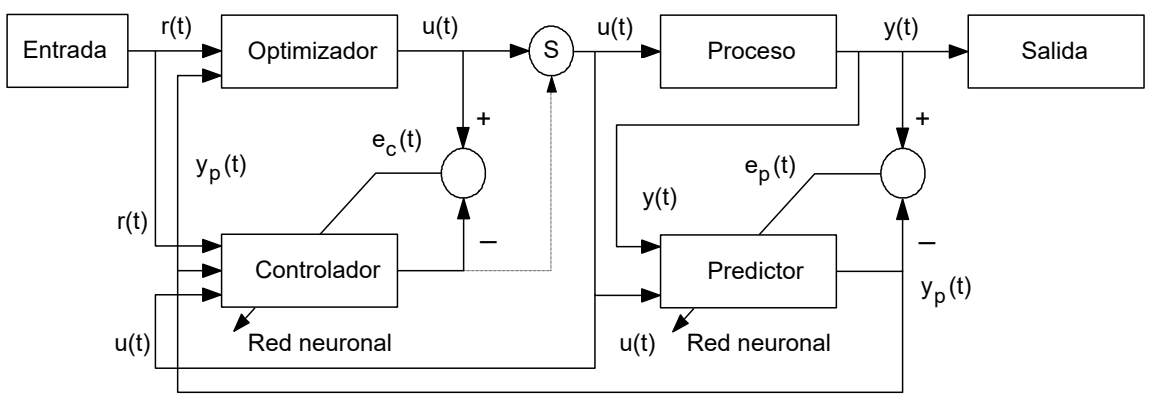

Figura 7. Diagrama en bloques sistema de control predictivo

Fuente: elaboración propia

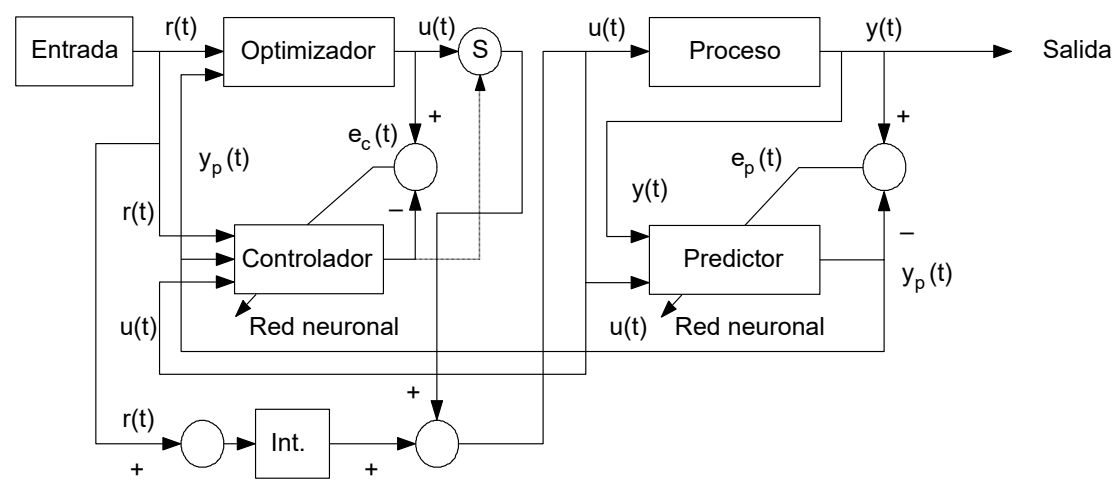

Figura 8. Esquema final del sistema de control desarrollado

Fuente: elaboración propia 


\section{DEFINICIÓN DE LA PLANTA}

Para la experimentación del sistema de control predictivo diseñado, y considerando que aún no se cuentan con datos de entrada/salida de la planta real a controlar, se ha optado por seleccionar un conjunto de sistemas (no lineales SISO y no lineal MIMO) que hayan servido anteriormente para ensayar otros sistemas de control predictivo o adaptativo $[8,17]$.

- Procesos no lineales (SISO)

$$
\begin{array}{ll}
y_{p}(k+1)=\frac{y_{p}(k)}{1+y_{p}^{2}(k)}+u^{2}(k) & 1^{\text {er }} . \text { Orden } \\
y_{p}(k+1)=\frac{y_{p}(k) y_{p}(k-1)\left[y_{p}(k)+2.5\right]}{1+y_{p}^{2}(k)+y_{p}^{2}(k-1)}+u(k) & 2^{\mathrm{do}} . \text { Orden }
\end{array}
$$

- Proceso no lineal (MIMO)

$$
\left[\begin{array}{l}
y_{p 1}(k+1) \\
y_{p 2}(k+1)
\end{array}\right]=\left[\begin{array}{c}
\frac{y_{p 1}(k)}{1+y_{p 2}^{2}(k)} \\
\frac{y_{p 1}(k) y_{p 2}(k)}{1+y_{p 2}^{2}(k)}
\end{array}\right]+\left[\begin{array}{l}
u_{1}(k) \\
u_{2}(k)
\end{array}\right]
$$

\section{RESULTADOS}

Por razones de espacio se muestra solo una parte de la gran cantidad de resultados obtenidos en las pruebas de simulación realizadas al sistema de control predictivo desarrollado. Se hicieron pruebas con diferentes señales de referencia, cambios en la ganancia y el momento del controlador y cambios en los parámetros de la red de predicción.

- Sistema SISO no lineal de primer orden:

$$
y_{p}(k+1)=\frac{y_{p}(k)}{1+y_{p}^{2}(k)}+u^{2}(k)
$$

En esta prueba, la entrada de referencia es una señal sinusoidal (figura 9) para simular cambios continuos en la referencia. La salida presenta ciertas diferencias respecto de la entrada en los cruces por cero. Posteriormente se realizaron algunos cambios en la ganancia y momento del controlador para corregir estas anomalías. 


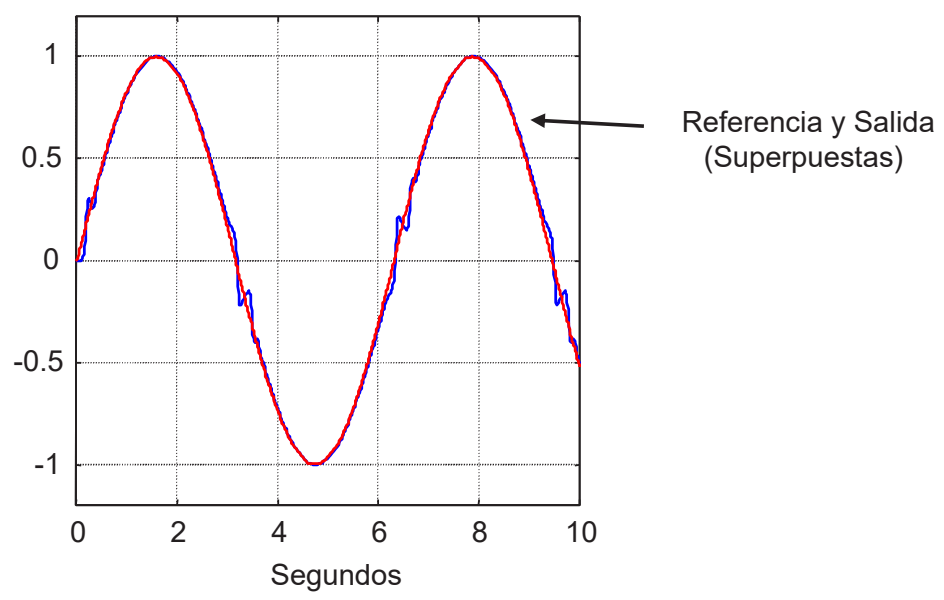

Figura 9. Referencia y salida sistema SISO no lineal de primer orden Fuente: elaboración propia

- Sistema SISO no lineal de segundo orden:

$$
y_{p}(k+1)=\frac{y_{p}(k) y_{p}(k-1)\left[y_{p}(k)+2.5\right]}{1+y_{p}^{2}(k)+y_{p}^{2}(k-1)}+u(k)
$$

Como se puede observar en las figuras 10 y 11, para el caso de control del sistema no lineal de segundo orden, efectuados los debidos ajustes, prácticamente las señales de entrada de referencia y salida quedan superpuestas observándose errores del orden de $10^{-6}$. En la figura 12 se presenta la salida a cambios bruscos en la entrada de referencia. La salida presenta una respuesta casi de ganancia infinita sin sobreimpulsos. Control sistema MIMO (multivariable) no lineal:

$$
\left[\begin{array}{l}
y_{p 1}(k+1) \\
y_{p 2}(k+1)
\end{array}\right]=\left[\begin{array}{c}
\frac{y_{p 1}(k)}{1+y_{p 2}^{2}(k)} \\
\frac{y_{p 1}(k) y_{p 2}(k)}{1+y_{p 2}^{2}(k)}
\end{array}\right]+\left[\begin{array}{l}
u_{1}(k) \\
u_{2}(k)
\end{array}\right]
$$

Por último, la figura 13 presenta los resultados logrados al controlar un sistema no lineal MIMO de dos entradas y dos salidas. Las señales de consigna, corresponden a una señal cuadrada y una señal sinusoidal. En la figura prácticamente no se logra apreciar nítidamente la diferencia entre las entradas y salidas, evidenciándose la calidad del control del sistema diseñado. 


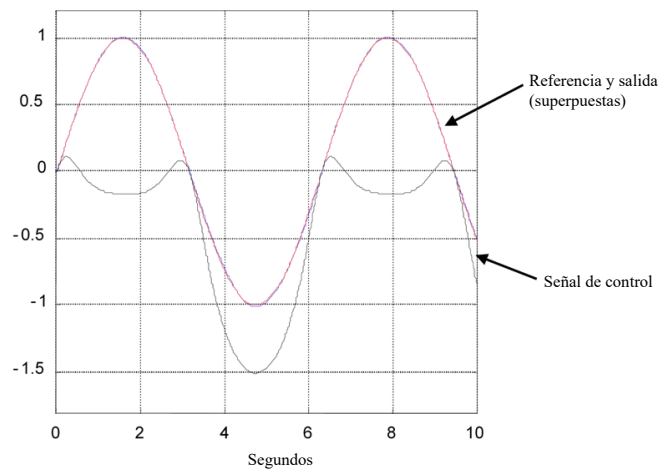

Figura 10. Referencia, salida y señal de control, sistema SISO no lineal de segundo orden Fuente: elaboración propia

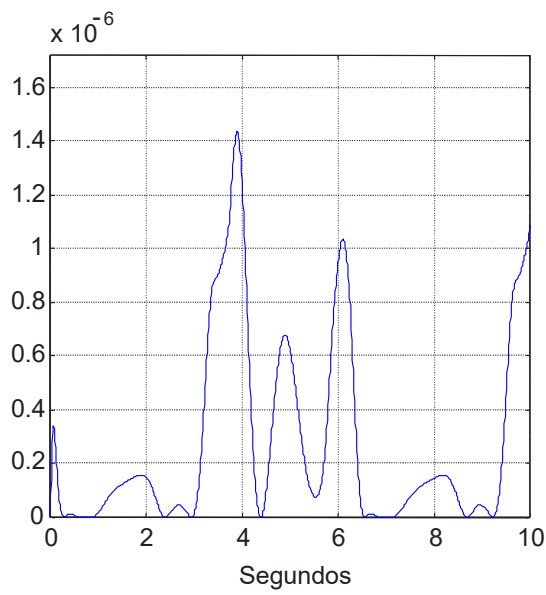

Figura 11. Error cuadrático medio, control sistema SISO no lineal de segundo orden. Fuente: elaboración propia

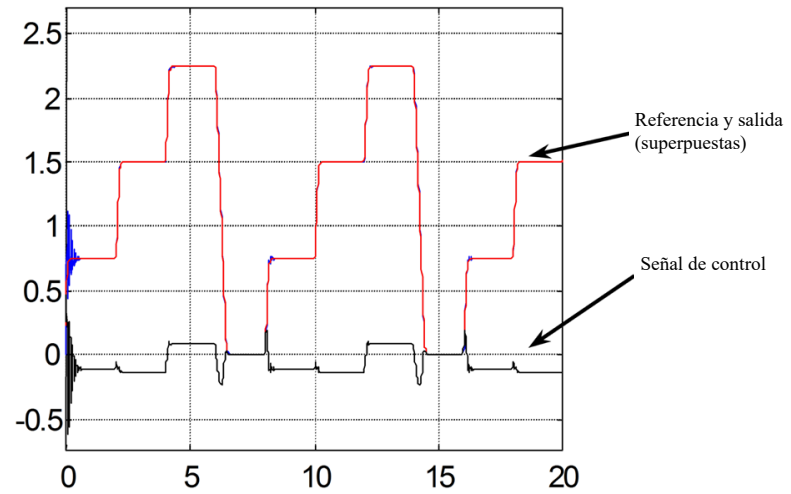

Figura 12. Referencia, salida y señal de control, sistema no lineal de segundo orden Fuente: elaboración propia 


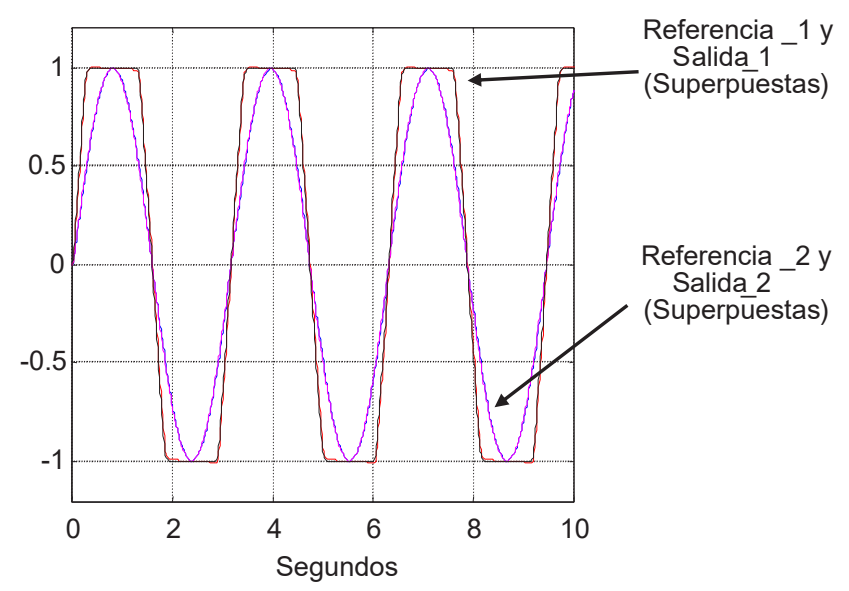

Figura 13. Referencias y salidas, control sistema MIMO no lineal Fuente: elaboración propia

\section{CONCLUSIONES Y TRABAJO FUTURO}

Se ha presentado el diseño y desarrollo de un sistema de control predictivo/adaptativo robusto, óptimo y eficiente, por cuanto el sistema trabaja con dos tipos de controladores, un optimizador como controlador primario y una red neuronal controladora como controlador secundario o de respaldo. La complejidad de los cálculos que realiza el optimizador dependen de la función de coste y de la topología de la red de predicción, sin embargo, luego de entrenada la red controladora con las señales provenientes del optimizador, esta desempeña idéntica función con igual desempeño y un menor coste de computación.

Es importante destacar además que, dado que la red de predicción que modela el proceso queda entrenando en línea, el sistema de control implementado también tiene el carácter de adaptativo lo cual significa que permanentemente se actualizará el modelo ante cambios de los parámetros de la planta si es que ello se produce.

El objetivo del sistema desarrollado es suplir algunas falencias en los sistemas convencionales utilizados previamente en el control de temperatura y nivel del estanque cónico en estudio.

Los resultados obtenidos en las pruebas de simulación han sido contrastados con resultados obtenidos en otros trabajos de investigación en los cuales se controlaron los mismos sistemas. Se constata que el esquema propuesto permite controlar de mejor manera estos sistemas, minimizando el periodo transitorio en la respuesta del sistema y el error de estado estacionario. El error cuadrático medio de seguimiento que se obtuvo en la mayoría de las pruebas efectuadas fue del orden de $10^{-6}$. 
Finalmente, y como trabajo futuro, queda ensayar el esquema de control predictivo desarrollado en el control de temperatura y nivel en la planta cónica no lineal en estudio, identificando previamente el modelo de la planta. Los resultados logrados en las pruebas de simulación permiten inferir que el esquema desarrollado, tendrá un buen desempeño en la planta real a controlar.

\section{REFERENCIAS}

[1] A. Conradie, C. Aldrich, "Neurocontrol of a multi-effect batch distillation pilot plant based on evolutionary reinforcement learning," Chemical Engineering Science, vol. 65, N. 5 , pp. 1627-1643, 2010.

[2] M. Bazaraa, H. Sherali, C.M. Shetty, Nonlinear programming: theory and Algorithms, 3. ${ }^{\mathrm{a}}$ ed., Nueva Jersey: Wiley Interscience, 2006, pp. 872.

[3] S. Chen, S. A. Billings, "Representations of non-linear systems: the NARMAX model," International Journal of Control, vol. 49, N. ${ }^{\circ}$ 3, pp. 1013-1032, 1988.

[4] H. González, M.S. Dutra, O. Lengerke, "Identification and modeling for non-linear dynamic system using neural networks type MLP," presentado en Proceedings of the 2009 Euro American Conference on Telematics and Information Systems: New Opportunities to increase Digital Citizenship, Praga, junio 03-05, 2009.

[5] R. Hecht-Nielsen, Neurocomputing, Boston: Ed. Addison Wesley, 1988, pp. 433.

[6] J. Vojtesek, P. Dostal, "Adaptive control of water level in real model of water tank, Process Control (PC)," presentado en 20th International Conference on, Strbske Pleso, Eslovaquia, junio 9-12, 2015.

[7] A. U. Levin y K. Narendra, "Control of nonlinear dynamical systems using neural networks," IEEE Neural Networks Council, vol.7, pp. 30-42, 1996.

[8] K. Narendra y K. Parthasarathy, "Identification and Control of Dynamical Systems Using Neural Networks," IEEE Transactions on Neural Networks, vol. 7, N. 1, 1996.

[9] H. M. Nguyen y N. Subbaram, "Advanced control strategies for wind energy systems: An overview", presentado en IEEE/PES Power Systems Conference and Exposition, Phoenix, 2011.

[10] K.J. Nidhil, S. Sreeraj, B. Vijay y V. Bagyaveereswaran, "System identification using artificial neural network", Circuit, Power and Computing Technologies (ICCPCT), presentado en 2015 International Conference, Nagercoil, 2015.

[11] M. Nørgaard, O. Ravn, NK. Poulsen y LK Hansen, Neural Networks for Modelling and Control of Dynamic Systems, Londres: Springer, 2000, pp. 246.

[12] K. Ogata, Ingeniería de control moderna, 4. a ed., Madrid: Prentice Hall, 2003, pp. 984. 
[13] D. T. Pham y L. Xing, Neural Networks for identification, prediction and control, Londres: Springer, 2012, pp. 238.

[14] A. Kupin, "Application of neurocontrol principles and classification optimisation in conditions of sophisticated technological processes of beneficiation complexes". Metallurgical y Mining Industry, vol. 6, pp. 16-24, 2014.

[15] R.J. Rajesh, R. Preethi, P. Mehata y B. Jaganatha Pandian, "Artificial neural network based inverse model control of a nonlinear process," presentado en Computer, Communication and Control (IC4), International Conference, Indore, 2015.

[16] V.R. Ravi, M. Monica, S. Amuthameena, S.K. Divya, S. Jayashree y J. Varshini, "Sliding Mode Controller for Two Conical Tank Interacting Level System," Applied Mechanics and Materials, vol. 573, pp. 273-278, 2014.

[17] A. M. Suárez, Nueva arquitectura de control predictivo para sistemas dinámicos no lineales usando redes neuronales, Tesis de Doctorado en Ciencias de la Ingeniería, Universidad de Chile, Santiago de Chile, 1998.

[18] D. Zhao, Z. Xia y D. Wang, "Model-Free Optimal Control for Affine Nonlinear Systems with Convergence Analysis", IEEE Transactions on Automation Science and Engineering, vol. 12, pp. 1461-1468, 2015. 